\title{
Influence of inpatient opioid consumption on persistent use following total knee arthroplasty
}

\author{
Meredith Pace (D) ,' Kerri Gannon, ${ }^{1}$ Matthew Friedland, ${ }^{1}$ Gervase Spurlin, ${ }^{1}$ \\ Lori Lyn Price ${ }^{2}$
}

'Anesthesiology, Lahey Hospital and Medical Center, Burlington, Massachusetts, USA

${ }^{2}$ Tufts Clinical and Translational Science Institute, Tufts University, Medford, Massachusetts, USA

Correspondence to Dr Meredith Pace, Anesthesiology, Lahey Hospital and Medical Center, Burlington, MA 01805, USA: meredith.pace@mac.com

Received 21 April 2020 Revised 7 August 2020 Accepted 11 August 2020 Published Online First 10 November 2020

\section{Linked}

- http://dx.doi.org/10.1136/ rapm-2020-102121

Check for updates

(C) American Society of Regional Anesthesia \& Pain Medicine 2021. No commercial re-use. See rights and permissions. Published by BMJ.

To cite: Pace M, Gannon K, Friedland $\mathrm{M}$, et al. Reg Anesth Pain Med 2021:46:99-103.

\section{ABSTRACT}

Background Total knee arthroplasty (TKA) is associated with a high incidence of continued opioid use beyond the expected period of recovery. The aim of this study was to determine the influence of inpatient opioid consumption on the development of persistent use.

Methods Opioid-naïve patients undergoing primary, uncomplicated TKA were included in a prospective, observational study at a single institution. Postoperatively, opioids were prescribed by orthopedic advanced practitioners or resident physicians and administered by registered nurses. Individual inpatient prescriptions were adjusted, as needed, to facilitate optimal recovery conditions. Average hourly inpatient opioid consumption was calculated from total usage between midnight on the day of surgery until the time of hospital discharge. Persistent opioid use was defined as a prescription refill written by the orthopedic surgeon at the 6-week surgical follow-up visit. A multivariable regression model was used to identify independent risk factors associated with persistent use.

Results A total of 351 patients were included in the analysis. Persistent opioid use was identified in $17.4 \%$ (61) of patients overall. A history of alcohol abuse (OR $7.80 ; 95 \% \mathrm{Cl} 2.13$ to $28.55, \mathrm{p}=0.002$ ) was identified as an independent risk factor. Inpatient opioid consumption in the top quartile (equivalent to more than $10 \mathrm{mg}$ of oxycodone every 4 hours) was not found to be associated with persistent use.

Conclusion Larger amounts of opioid consumed in the hospital following uncomplicated, primary TKA may not be associated with an increased risk of persistent use at 6 weeks among opioid-naïve patients. Patients may have unique risk factors for prolonged opioid use that may not necessarily be apparent in the early postoperative period.

\section{INTRODUCTION}

Patient requests for opioid prescription refills beyond the expected recovery time following uncomplicated surgery present a dilemma for prescribers. Continued opioid consumption after the period of normal postoperative healing has been referred to as persistent use. ${ }^{1}$ The incidence of new, persistent opioid use following major surgical procedures among patients over 65 years old may be as high as 1 in $10 .^{2}$ Older adults who consume opioids are at an increased risk of falls and fractures, motor vehicle accidents, cardiac and respiratory events, as well as cognitive decline. ${ }^{3}$ Age-related changes in opioid pharmacokinetics and pharmacodynamics are responsible for this increased risk of adverse effects, especially when opioids are combined with other agents that affect the central nervous system. ${ }^{4}$

Persistent opioid use following total knee arthroplasty (TKA) occurs despite improvements in surgical joint pain, suggesting this is an indication of misuse. ${ }^{5}$ The incidence of persistent opioid use following TKA varies depending on the definition used, but it is among the highest when compared with other major surgical procedures. ${ }^{5-8}$ The demand for TKA is rising as the population ages in the USA. The number of annual cases is projected to reach 3.48 million by $2030 . .^{9}$ Considering this evidence, risk mitigation strategies that effectively reduce the rate of persistent opioid use following TKA can have a profound impact on public health, especially among the elderly.

Larger amounts of opioid prescribed to patients at the time of hospital discharge have been associated with new, persistent opioid use following many common surgical procedures. ${ }^{10-13}$ These studies suggest that limiting postoperative prescribing can effectively reduce risk among opioid-naïve patients. However, we currently do not know if the amount of opioid consumed by patients recovering in the hospital also plays a role in the development of persistent opioid use. This knowledge could have important practice implications for providers who assess and treat acute, postoperative pain among inpatients. Opioid titration is a careful balance between optimizing a patient's ability to effectively rest and participate in physical therapy while minimizing the risk of adverse drug effects, such as the development of persistent use. To our knowledge, this study is the first to investigate a relationship between inpatient opioid consumption and persistent use.

We evaluated the association between the amount of opioid consumed during the inpatient period and the development of persistent use beyond the expected period of recovery after TKA among opioid-naïve patients. We defined the expected period of recovery as 6 weeks. We hypothesized that those patients who consumed the largest amount of opioid in the hospital would be more likely to become persistent users.

\section{METHODS}

Patient population

Subject enrollment and data collection occurred over a 2-year period from September 2017 to June 2019. Surgeries were performed by five fellowshiptrained orthopedic surgeons at Lahey Hospital and 
Medical Center (LHMC) and all patients were admitted to the hospital postoperatively.

Patients over the age of 18 years old who had undergone TKA were screened for study eligibility using the following criteria. Patients whose procedure was designated as 'revision', 'complex' or 'bilateral' by surgical case coding were not included. Only patients who were opioid-naïve at the time of surgery were offered enrollment. Preoperative opioid use was assessed by a review of current medications obtained from each patient's electronic medical record (EMR). This list was updated with each patient at the preoperative clinic visit and again with nursing staff on the day of surgery to ensure inclusion of all medications prescribed by both LHMC and outside providers. Preoperative clinic visits are a requirement for all patients scheduled to undergo total joint arthroplasty at LHMC. Patients without a documented active opioid prescription (including tramadol and codeine) on the day of surgery and who verbally confirmed that they were not using an opioid at the time of surgery were considered 'opioid-naïve'. This definition is consistent with similar studies. ${ }^{5} 14$ Patients with a documented active opioid prescription or who verbally confirmed taking an opioid at the time of surgery were excluded. Patients with a history of chronic opioid use, defined as use on most days for greater than a 3-month period, ${ }^{15}$ or a history of an opioid use disorder were excluded. Patients with a history of illicit drug abuse or polysubstance abuse were also excluded.

Eligible patients were offered participation in the study following surgery and prior to discharge from the hospital. Written informed consent was obtained from all study participants.

\section{Perioperative care}

A standardized, evidence-based perioperative pathway ${ }^{16}$ is in place for patients presenting for TKA at LHMC. Optimization of postoperative analgesia is a major component of these protocols. Preoperatively, patients without contraindications receive a dose of acetaminophen and a non-steroidal anti-inflammatory (NSAID). Unless contraindicated, all patients receive an adductor canal block. Neuraxial anesthesia with a spinal block is preferred over general anesthesia unless a patient-specific contraindication exists. A postoperative analgesic regimen includes scheduled NSAIDs and acetaminophen, except when a patient has a known contraindication to either class of medication. An inpatient prescription for oxycodone $5 \mathrm{mg}$, one or two tablets, is available every 4 hours, as needed. An additional $0.2 \mathrm{mg}$ of intravenous hydromorphone is also available every 4 hours to treat breakthrough pain during the first 24 hours following surgery. Prescribed opioids are dispensed to inpatients at the discretion of registered nurses who choose an appropriate strength, frequency and route of administration based on clinical factors, including patient-reported pain scores, functional status and evidence of side effects. Orthopedic advanced practitioners and resident physicians evaluate inpatients regularly and adjust opioid prescriptions based on patients' subjective response, functional status and nursing input.

Following hospital discharge, patients are seen at follow-up visits with their orthopedic surgeon at approximately 2 and 6 weeks. Patients who request additional opioids for the treatment of ongoing surgical site pain are given a prescription by the orthopedic surgeon. Referral to a pain specialist is not routine practice. Subsequent follow-up visits are made at the discretion of the orthopedic surgeon and may be independently staffed by an advanced practitioner.

\section{Data collection}

Patient factors including age, gender, race and American Society of Anesthesiologists' physical classification (ASA) scores were obtained from each subject's EMR. The length of hospital stays and disposition to home versus a rehabilitation facility were recorded. All documented opioid doses were converted to oral morphine milligram equivalents (MME) using a standard opioid conversion chart. ${ }^{17}$ Total inpatient opioid administration, including medication type, strength and route of administration was recorded from midnight on the day of surgery until the time of hospital discharge. An average hourly opioid dose was calculated for each subject. Discharge opioid prescription details, including medication type, pill strength and quantity dispensed were obtained from the discharge summary and a total dose was calculated for each subject.

Selected risk factors for persistent postsurgical opioid use were chosen based on associations established by similar investigations. ${ }^{1618}$ Each subject's EMR was assessed for the following selected risk factors. A history of alcohol abuse was recorded, as was a current prescription for an antidepressant, a benzodiazepine or active tobacco or marijuana use. In the most cases, this information had been entered into the EMR by a patient's primary care provider. Any active medications or diagnoses provided by outside providers were added to a patient's EMR by preoperative clinic staff. Postoperative opioid consumption exceeding the top quartile in our sample was chosen based on the findings of multiple previous studies suggesting this is also a risk factor for the development of persistent opioid use following surgery. ${ }^{101213}$

All medical encounters and prescriptions were reviewed from the day of hospital discharge to the 6 weeks surgical follow-up visit in each subject's EMR. Patients who experienced medical or surgical complications were withdrawn, as were patients who had additional surgery within 6 weeks following TKA. Persistent opioid use was defined as a prescription refill written by the orthopedic surgeon at the 6 weeks surgical follow-up visit.

\section{Statistical analysis}

All analyzes were conducted using SAS Enterprise V.7.15. Univariate analyzes were used to examine relationships between each individual risk factor and opioid use at 6 weeks. Length of stay was analyzed as a continuous variable while all other variables were analyzed as categorical data. Continuous data were assessed for normality and the analysis of variance or Wilcoxonrank sum test were used as appropriate. Categorical variables were analyzed via the $\chi^{2}$ test. $P$ values were two tailed, and significance was set at $\mathrm{p}<0.05$. Backward selection was used to develop a multivariable regression model that retained variables reaching significance by univariate analysis. Candidate variables for the backward selection were age, sex, length of stay, ASA score, disposition to a rehabilitation facility, active antidepressant prescription, active benzodiazepine prescription, history of alcohol abuse, active tobacco or marijuana use, top quartile for average hourly inpatient opioid consumption, and top quartile for discharge prescription size.

\section{RESULTS}

Between 2017 and 2019, a total of 441 patients who had successfully undergone TKA at LHMC were screened for study eligibility (figure 1). A total of 55 patients were excluded from enrollment due to opioid use at the time of surgery, a history of chronic opioid use or a history of illicit drug abuse. Thirteen patients refused to participate. Twenty-two subjects were 


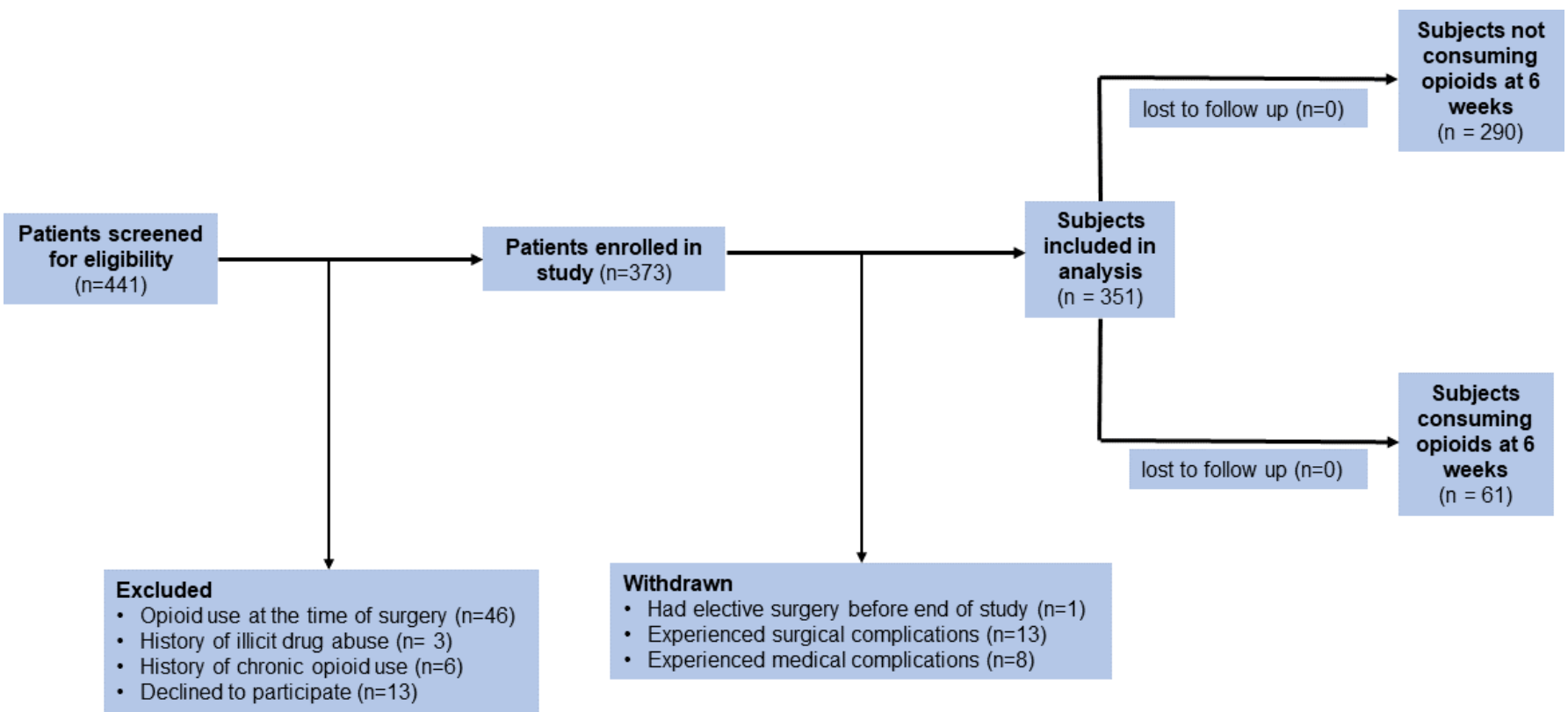

Figure 1 STROBE study diagram. STROBE, Strengthening the Reporting of Observational Studies in Epidemiology.

withdrawn after enrollment due to medical or surgical complications, or they had undergone additional surgery within 6 weeks of the index surgery. Surgical complications included joint contracture requiring manipulation, wound complications, joint space infections and femoral condyle fracture or dislocation. Medical complications included bowel perforation, gastric bleed, unstable atrial fibrillation, and symptomatic kidney stones. No remaining subjects received additional opioid prescriptions from providers other than the LHMC orthopedic service during the study period.

A total of 351 subjects were included in our analysis (table 1 ). The mean subject age was 68 years and $60.4 \%$ were female. Most

Table 1 Characteristics and perioperative variables between patients who stopped or continued to use opioids 6 weeks following total knee arthroplasty

\begin{tabular}{|c|c|c|c|}
\hline & Not using opioid at 6 weeks & Using opioid at 6 weeks & $P$ value \\
\hline Sample size, $\mathrm{n}(\%)$ & $290(82.6)$ & $61(17.4)$ & \\
\hline \multicolumn{4}{|l|}{ Sociodemographic characteristics } \\
\hline Sex, $n(\%)$ & & & 0.96 \\
\hline Female & $175(60.4)$ & $37(60.7)$ & \\
\hline Male & $115(39.7)$ & $24(39.3)$ & \\
\hline Black & $3(1.0)$ & $0(0.0)$ & \\
\hline Other & $12(4.2)$ & $1(1.6)$ & \\
\hline \multicolumn{4}{|l|}{ Comorbidities } \\
\hline ASA classification, $\mathrm{n}(\%)$ & & & $0.03 *$ \\
\hline 1 or 2 & $181(62.4)$ & $29(47.5)$ & \\
\hline History of alcohol abuse, $\mathrm{n}(\%)$ & $4(1.4)$ & $6(9.8)$ & $<0.01^{*}$ \\
\hline Active tobacco or marijuana use, $\mathrm{n}(\%)$ & $15(5.2)$ & $7(11.5)$ & 0.72 \\
\hline \multicolumn{4}{|l|}{ Hospital course } \\
\hline Median length of stay (IQR) in days & 3 (2 to 3 ) & $3(2$ to 3$)$ & 0.10 \\
\hline Disposition, $\mathrm{n}(\%)$ & & & 0.86 \\
\hline Home & $245(84.5)$ & $51(83.6)$ & \\
\hline Rehabilitation facility & $45(15.5)$ & $10(16.4)$ & \\
\hline Top quartile for average hourly inpatient opioid consumption, n (\%) & $66(22.8)$ & $20(32.8)$ & 0.10 \\
\hline Top quartile for discharge prescription size, $n(\%)$ & $67(23.1$ & $16(26.2)$ & 0.60 \\
\hline
\end{tabular}

*Indicates statistical significance $(p<0.05)$.

ASA, American Society of Anesthesiologists. 
Table 2 Distribution of inpatient opioid consumption and initial prescription size

\begin{tabular}{lrrrrrrr}
\hline & Mean & \multicolumn{1}{c}{ SD } & Minimum & 25th percentile & Median & 75th percentile & Maximum \\
\hline Average hourly inpatient opioid consumption & 2.4 & 1.6 & 0.0 & 1.1 & 2.2 & 3.4 & 7.8 \\
Initial prescription size & 387.0 & 194.8 & 0.0 & 240.0 & 375.0 & 450.0 & 1275.0 \\
\hline
\end{tabular}

Values given in oral morphine milligram equivalents.

subjects were white (95.4\%) and $0.9 \%$ were black. The median length of hospital stay was 3 days and $15.7 \%$ were discharged to a rehabilitation facility rather than home. Forty per cent of subjects were assigned an ASA score of 3 or 4 (vs a score of 1 or 2) and $2.9 \%$ had a history of alcohol abuse. At the time of surgery, $22.2 \%$ of subjects had a prescription for an antidepressant and $15 \%$ had a prescription for a benzodiazepine. Active tobacco or marijuana use was recorded in $6.3 \%$ of subjects. The overall rate of persistent opioid use was $17.4 \%$ (61 of 351).

The distribution of inpatient opioid consumption and initial prescription size is presented in table 2. The top quartile for average hourly inpatient opioid consumption was calculated to be greater than 3.4 MME per hour. For clinical reference, this is equivalent to greater than $82 \mathrm{MME}$ per day or $10 \mathrm{mg}$ of oxycodone every 4 hours. The top quartile for initial opioid prescription size was calculated to be greater than $450.0 \mathrm{MME}$. This is equivalent to more than 60 tablets of $5 \mathrm{mg}$ oxycodone tablets.

Univariate analysis demonstrated that a higher ASA score of 3 or 4 (reference value ASA score of 1 or 2) and a history of alcohol abuse had a significant association with persistent opioid use. However, the top quartiles of average hourly inpatient opioid consumption and initial prescription size did not show a statistically significant association with persistent opioid use. Likewise, discharge to a rehabilitation facility and length of hospital stay were not found to be significantly associated with the primary outcome in our sample. Only a history of alcohol abuse retained significance in the multivariable model (OR 7.80; 95\% CI 2.13 to $28.55, \mathrm{p}=0.002)$. Results were similar when age, sex and race were included in the multivariable model.

\section{DISCUSSION}

We did not find a significant association between inpatient opioid consumption and persistent opioid use following primary, uncomplicated TKA. These results could have important implications for those opioid-naïve patients who require larger daily amounts of opioid in the initial days following major orthopedic surgery. For such patients, limiting opioids to a standard inpatient prescription size runs the risk of providing inadequate pain control and suboptimal recovery conditions. Although the risk of other opioid-related adverse events must be considered, judicious titration to meet greater individual inpatient requirements may not increase the risk for developing persistent postoperative opioid use after hospital discharge.

Our definition of persistent of opioid use begins much sooner after surgery than a 90-day postoperative date designated by similar studies. ${ }^{10-13}$ We defined persistent use after primary TKA as an opioid prescription written by the orthopedic surgeon at the 6-week follow-up visit. For most patients, this represents their third opioid refill and an event that is associated with a significantly increased probability of long-term use. In 2017, the US Centers for Disease Control and Prevention (CDC) evaluated characteristics of opioid prescriptions and their impact on the likelihood of long-term opioid use. ${ }^{19}$ One of the largest incremental increases in the probability of continued use was observed when a patient received a third prescription. As such, in contrast to the initial postoperative opioid prescription dispensed at the time of hospital discharge and refilled at the first surgical follow-up visit, additional opioid prescriptions provided at subsequent encounters have the potential to confer more risk than benefit to patients.

To investigate a relationship between inpatient opioid consumption and persistent use, we were required to review each subject's EMR. By doing so, we were able to accurately record each dose of opioid administered in the hospital. In reviewing each clinical encounter that occurred in the 6 weeks following the index surgery, we were able to accurately identify patients who experienced postoperative complications or subsequent surgical procedures that could have significantly influenced the duration of opioid consumption. We were also able to identify any opioid prescriptions not provided by the orthopedic surgical service.

Our study included only subjects having the same elective procedure at a single institution by a limited number of surgeons. This design naturally limited error that may have been introduced by differences in preoperative treatment, surgical and anesthetic techniques, as well as postoperative care. This allowed us to minimize the variability in severity and duration of acute, post-surgical pain which has the potential to influence the amount and duration of opioid use.

\section{Limitations}

Our study should be interpreted cautiously considering its limitations. First, our study is comprised of observational data which has the inherent risk of confounding by unmeasured variables. We did not analyze a comprehensive list of risk factors known to be associated with chronic opioid use in the general medical population, but rather chose variables with the highest adjusted risk calculated by recent investigations of persistent postoperative opioid use. ${ }^{1618}$ Interestingly, we did not find similar associations with active tobacco, antidepressant or benzodiazepine use among our cohort of patients. We also did not find an association between the top quartile of initial opioid prescription size and persistent use. This may be explained, in part, by the timing of our data collection. In 2016 when the CDC published a guideline for prescribing opioids, ${ }^{15}$ there was a growing focus on the developing opioid crisis in the USA which prompted efforts to reduce opioid utilization. By late 2017, when data collection for our study began, subsequent alterations in practice policy had taken place at many institutions like LHMC. Increased attention to limiting opioid prescription sizes dispensed at the time of hospital discharge may explain why the lower limit of our top quartile for initial prescription size was only $450 \mathrm{MME}$. Initial prescription sizes of greater than $700 \mathrm{MME}$ have been found by the CDC to be associated with an increased probability of prolonged opioid use. ${ }^{19}$

Our internal review board did not permit query of the state online prescription drug monitoring program for research purposes. Opioid prescriptions dispensed to subjects by providers outside of LHMC during the perioperative period may not have been captured unless reported by a subject. Lastly, all opioid prescriptions written for subjects during the perioperative period were assumed to be for the purposes of personal use. 
Alternative intentions for opioid prescriptions, such as diversion, would not have been detected by our data collection methods.

\section{Conclusion}

At our institution, approximately one in six previously opioidnaïve patients continued to use opioids at 6 weeks after uncomplicated, primary TKA, possibly representing inappropriate use. A history of alcohol abuse was found to be associated with significantly higher odds of persistent use, by our definition. However, subjects who consumed an average hourly amount in the top quartile of our cohort as inpatients were not more likely to continue opioid use beyond the expected period of recovery. Our findings suggest that restricting inpatient opioid prescriptions to an equivalent of 10 milligrams of oxycodone every 4 hours after major orthopedic surgery may not effectively mitigate the risk of persistent postoperative use. Such prescribing limits run the risk of denying some patients appropriate treatment for postsurgical pain.

\section{Twitter Matthew Friedland @mattfriedland}

Acknowledgements Adult Reconstructive Surgery Program, Department of Orthopedic Surgery, Lahey Hospital and Medical Center, Burlington, Massachusetts, USA.

Contributors MP, KG and MF designed and planned the study. MP, KG, MF and GS helped with data collection. LLP provided statistical expertise in study design and conducted the statistical analysis. MP wrote the manuscript. KG, MF and LLP helped review and edit the manuscript. All authors contributed to the approval of the final manuscript.

Funding The project described was supported by the National Center for Advancing Translational Science, National Institutes of Health, Award Number UL1TR002544.

Disclaimer The content is solely the responsibility of the authors and does not necessarily represent the views of the NIH.

Competing interests None declared.

Patient consent for publication Not required.

Ethics approval This prospective, single-center observational study was approved by the institutional review board at Lahey Hospital and Medical Center (LHMC).

Provenance and peer review Not commissioned; externally peer reviewed.

Data availability statement All data relevant to the study are included in the article or uploaded as online supplemental information.

ORCID iD

Meredith Pace http://orcid.org/0000-0003-2427-317X

\section{REFERENCES}

1 Brummett CM, Waljee JF, Goesling J, et al. New persistent opioid use after minor and major surgical procedures in US adults. JAMA Surg 2017;152:e170504.

2 Santosa KB, Hu H-M, Brummett CM, et al. New persistent opioid use among older patients following surgery: A Medicare claims analysis. Surgery 2020;167:732-42.

3 Naples JG, Gellad WF, Hanlon JT. Managing pain in older adults: the role of opioid analgesics. Clin Geriatr Med 2016;32:725-35.

4 By the 2019 American Geriatrics Society Beers Criteria ${ }^{\circledR}$ Update Expert Panel. American geriatrics Society 2019 updated AGS beers Criteria $\circledast$ for potentially inappropriate medication use in older adults. J Am Geriatr Soc 2019;67:674-94.

5 Goesling J, Moser SE, Zaidi B, et al. Trends and predictors of opioid use after total knee and total hip arthroplasty. Pain 2016:157:1259-65.

6 Sun EC, Darnall BD, Baker LC, et al. Incidence of and risk factors for chronic opioid use among opioid-naive patients in the postoperative period. JAMA Intern Med 2016;176:1286-93.

7 Cook DJ, Kaskovich SW, Pirkle SC, et al. Benchmarks of duration and magnitude of opioid consumption after total hip and knee arthroplasty: a database analysis of 69,368 patients. J Arthroplasty 2019;34:638-44.

8 Sabatino MJ, Kunkel ST, Ramkumar DB, et al. Excess opioid medication and variation in prescribing patterns following common orthopaedic procedures. J Bone Joint Surg Am 2018:100:180-8.

9 Kurtz S, Ong K, Lau E, et al. Projections of primary and revision hip and knee arthroplasty in the United States from 2005 to 2030. J Bone Joint Surg Am 2007;89:780-5.

10 Brescia AA, Waljee JF, Hu HM, et al. Impact of prescribing on new persistent opioid use after cardiothoracic surgery. Ann Thorac Surg 2019;108:1107-13.

11 Gil JA, Gunaseelan V, DeFroda SF, et al. Risk of prolonged opioid use among opioidnaïve patients after common shoulder arthroscopy procedures. Am I Sports Med 2019;47:1043-50.

12 Gossett TD, Finney FT, Hu HM, et al. New persistent opioid use and associated risk factors following treatment of ankle fractures. Foot Ankle Int 2019;40:1043-51.

13 Finney FT, Gossett TD, Hu HM, et al. New persistent opioid use following common forefoot procedures for the treatment of hallux valgus. I Bone Joint Surg Am 2019;101:722-9.

14 Larach DB, Sahara MJ, As-Sanie S, et al. Patient factors associated with opioid consumption in the month following major surgery. Ann Surg 2019;Publish Ahead of Print.

15 Dowell D, Haegerich TM, Chou R. CDC Guideline for Prescribing Opioids for Chronic Pain--United States, 2016. JAMA 2016;315:1624-45.

16 Soffin EM, Gibbons MM, Ko CY, et al. Evidence review conducted for the agency for healthcare research and quality safety program for improving surgical care and recovery: focus on anesthesiology for total knee arthroplasty. Anesth Analg 2019;128:441-53.

17 Calculating total daily dose of opioids for safer dosage. Available: https://www.cdc. gov/drugoverdose/pdf/calculating_total_daily_dose-a.pdf

18 Politzer CS, Kildow BJ, Goltz DE, et al. Trends in opioid utilization before and after total knee arthroplasty. J Arthroplasty 2018;33:S147-53.

19 Shah A, Hayes CJ, Martin BC. Characteristics of Initial Prescription Episodes and Likelihood of Long-Term Opioid Use - United States, 2006-2015. MMWR Morb Mortal Wkly Rep 2017;66:265-9. 